\title{
EMPRESARIALISMO Y TRANFORMACIÓN URBANA. EL CASO DE LA CIUDAD DE MÉXICO'
}

\section{URBAN ENTREPRENEURIALISM IN MEXICO CITY. TRANSFORMATION OF THE CENTRAL DISTRICT}

\author{
Luis Alberto Salinas Arreortua \\ Instituto de Geografía, UNAM \\ Isalinas@igg.unam.mx
}

Resumen: El empresarialismo urbano constituye un proceso clave en las actuales transformaciones urbanas. Mediante el mismo las autoridades locales estimulan la inversión del capital privado en el contexto neoliberal. El presente trabajo baraja la vinculación de este concepto con el de gentrificación y lo aplica a las transformaciones recientes en el área central de la Ciudad de México. Se vale para ello de un estudio de caso, avalado por un extenso trabajo de campo, consistente en el levantamiento de usos del suelo y entrevistas en profundidad, combinado con diversas fuentes secundarias. Las transformaciones recientes de estos sectores céntricos muestran distintas características de la gestión empresarialista de la política, tales como la estimulación de la inversión privada como principal motor de la recualificación urbana.

Palabras clave: empresarialismo urbano, Ciudad de México, autoridades locales, inversión de capital.
Abstract: Urban entrepreneurialism constitutes a key process in current urban transformations. By these means, local authorities encourage investments of private capital in the neoliberal context. The present work links this concept with the process of gentrification and applies both of them to recent transformations in the central area of Mexico City. It relies on a case study, backed up by extensive field work, in which surveys of land uses and qualitative interviews are combined with diverse secondary sources. Current transformations in the central district show distinct characteristics of urban entrepreneurialism, such as the stimulation of private investment as the main factor in urban requalification.

Key Word: urban entrepreneurialism, Mexico City, local authorities, capital investment

1 Este trabajo se elaboró durante una estancia de investigación posdoctoral en el Departamento de Ciencia Política y Relaciones Internacionales, Universidad Autónoma de Madrid. El autor agradece al proyecto de investigación Contested_Cities y a la Secretaría de Ciencia, Tecnología e Innovación del Distrito Federal, México por la beca otorgada para la realización de la estancia de investigación. 


\section{Introducción}

La transición de la metrópolis desarrollista de mediados del siglo XX hasta la metrópolis neoliberal iniciada hacia el último cuarto del mismo siglo han propiciado diversos procesos urbanos, los cuales se han definido de distintas maneras: segregación, gentrificación, expansión urbana, policentrismo, por mencionar algunos. Al mismo tiempo, desde la gestión urbana, se pueden identificar tendencias en común que han caracterizado las transformaciones urbanas en el contexto neoliberal, conocidas bajo el concepto de empresarialismo urbano (Harvey, 1989 y 2001). Una de las características del empresarialismo urbano es la alianza entre el sector público y el sector privado, lo que ha generado, entre otros aspectos, el retorno de capital a los centros históricos y áreas centrales de las grandes ciudades, provocando el proceso conocido como gentrificación.

El objetivo principal de este trabajo es, a partir de la noción conceptual de "empresarialismo urbano" (Harvey, 1989 y 2001), identificar los elementos que caracterizan la gestión urbana del gobierno local. El objetivo de estas prácticas consiste en estimular la inversión privada en las áreas centrales: políticas públicas encaminadas a erradicación de sectores de población de escasos recursos, incluida población indigente y vendedores ambulantes; interés por realizar intervenciones puntuales en el lugar y no en el desarrollo territorial, y políticas fiscales para favorecer la inversión privada. Todas estas acciones constituyen la forma de participación del gobierno de la Ciudad de México (Gobierno del Distrito Federal) y de las distintas Delegaciones (municipios) que integran el Distrito Federal, ante los procesos de transformación urbana.

Este análisis del empresarialismo urbano se aplica al caso de estudio de la colonia Condesa, barrio localizado en el área central de la Ciudad de México. Aquí, la gestión urbana del gobierno de la Ciudad de México y de la Delegación Cuauhtémoc (jurisdicción administrativa a la que pertenece dicha colonia) han contribuido a impulsar diversas transformaciones urbanas, las cuales consisten principalmente en: incremento de establecimientos comerciales a partir de cambios de uso de suelo y de cambios de giro mercantil, y crecimiento de oferta inmobiliaria de uso habitacional dirigida para un sector de ingresos medios y altos. Dichas transformaciones han sido identificadas previamente con el concepto de gentrificación (Salinas, 2014).

Para realizar esta investigación, una vez analizado el concepto de empresarialismo urbano, se destacan las principales transformaciones urbanas que se están produciendo en la colonia Condesa. Para conocer el crecimiento de establecimientos comerciales se realizó un levantamiento de uso de suelo, durante el primer semestre del 2011, de cada uno de los 1341 predios localizados en la colonia Condesa. Por otro lado, se recurrió a solicitar información con base en la Ley de Transparencia y Acceso a la Información Pública del Distrito Federal a distintas dependencias del gobierno local, tanto del Gobierno del Distrito Federal como del gobierno de la Delegación Cuauhtémoc. Respecto de la dinámica del sector inmobiliario, se obtuvo información a través del sitio electrónico especializado metroscubicos, sitio electrónico que constituye un referente para conocer la oferta de vivienda actual. También se consultaron diversos estudios realizados en este sitio sobre el comportamiento inmobiliario. La información sobre establecimientos comerciales y la del sector inmobiliario se complementaron con 38 entrevistas semiestructuradas, dirigidas a autoridades del gobierno del Distrito Federal y de la Delegación Cuauhtémoc, dueños y responsables de establecimientos comerciales y representantes del sector inmobiliario y vecinos de la colonia. 


\section{Reestructuración económica neoliberal}

Ha sido amplia la discusión teórica y empírica de los cambios ocurridos en el proceso productivo de acumulación global del capital y sus distintas repercusiones territoriales en diversas partes del mundo (Harvey, 1990; Amin y Robins, 1992; Boyer, 1992; Ramírez y Hauser, 1996; Méndez, 1997; De Mattos, 2002; Mertins, 2005; Duhau y Giglia, 2008; Portes y Roberts, 2008; Ciccolella y Mignaqui, 2009; Pradilla, 2009). Nos referimos a los cambios presentados a finales de los años setenta del siglo XX en el régimen de acumulación del capital, al pasar de una intervención estatal y un proceso productivo fordista a coexistir con el modelo de acumulación flexible regulado a partir de las políticas neoliberales.

Diversas son las políticas que caracterizan al periodo neoliberal, algunas de las cuales son: la privatización de empresas públicas, permitir la inversión privada en las actividades económicas que anteriormente realizaba el Estado e incluso en los sectores estratégicos, eliminación gradual de los aranceles a la importación, firma de diversos tratados comerciales, reducción de la inversión pública además de implementar diversos incentivos fiscales para la atracción de capital privado, privatización de servicios, descentralización de la administración pública brindando mayor autonomía a los gobiernos locales, entre otras.

Este giro neoliberal, aunado a un desarrollo tecnológico basado en las tecnologías de la información y comunicaciones, ha modificado tanto la forma de producción como la organización de las empresas, impactando de manera significativa en la transformación de las grandes ciudades. Esta reestructuración económica, en el ámbito territorial, se caracteriza por acelerar un proceso de desconcentración industrial y poblacional hacia la periferia metropolitana, continuando con un proceso de expansión urbana metropolitana y regional. Aunado a esta doble desconcentración, se produce una nueva concentración o recentralización de nuevas y diversas actividades que han crecido en los últimos años, en particular de servicios al productor (servicios financieros y de seguros, inmobiliarios, profesionales legales y de contabilidad, científicos y técnicos, principalmente) en las principales ciudades, es decir, una recentralización con desconcentración (Hiernaux, 1998: 113), mientras que en el interior de las grandes ciudades, se genera una dispersión de estas actividades económicas, transformando la estructura intraurbana, al pasar de una funcionalidad monocéntrica a una policéntrica (Aguilar, 2002: 7; Goulart, 2005: 1), situación que caracteriza a las grandes metrópolis latinoamericanas.

La reestructuración económica ha implicado diversas transformaciones en el patrón de urbanización. Si en el periodo de Industrialización por Sustitución de Importaciones (ISI) se caracterizó por una marcada expansión periférica, en la actualidad, además de las diversas construcciones en la periferia, existe una fuerte tendencia de construir y regresar hacia la ciudad existente (Carrión, 2004: 35). Bajo este escenario, las áreas centrales de las grandes ciudades, se convierten en un espacio en disputa, es decir, espacios con “...diversos grupos de interés divergentes: casas comerciales tradicionales, inversionistas, residentes, dueños y corredores de bienes raíces, comerciantes ambulantes, políticos, planificadores, etcétera." (Herzog, 2004: 268).

El interés por la ciudad existente se hace evidente en la dinámica del capital financiero y en general el sector servicios, desplazando actividad industrial de las áreas centrales. Además, el capital inmobiliario se ha convertido en uno de los principales responsables en la transformación del territorio en las últimas décadas (Monroy y 
Martínez, 2008: 2). Importante es mencionar que todo ha sido posible a partir del rol que han jugado los gobiernos locales en el contexto neoliberal.

\section{Empresarialismo urbano}

Hacia finales de los ochenta, derivado de los problemas económicos y presupuestarios de muchas ciudades en todo el mundo a partir de la recesión de 1973, David Harvey refiere la organización de un coloquio en Orleans en 1985 en el cual académicos, empresarios y políticos llegaron a un consenso: que los gobiernos urbanos tenían que ser mucho más innovadores y emprendedores. A partir de ese momento parece haber emergido un consenso en general en que para las ciudades será ventajoso adoptar una actitud empresarial respecto al desarrollo económico (Harvey, 2001: 167-368).

Ante el cambio del régimen de acumulación de capital, cuya reestructuración territorial del espacio urbano produjo una recentralización con desconcentración, junto a la aplicación de políticas neoliberales tales como la reducción de la participación del Estado en la economía, lo cual implica entre otros factores, austeridad presupuestal y disminución de gasto social de los distintos niveles de gobierno y (des)regulación del mercado, se aprecia una tendencia de la aplicación del empresarialismo como forma de gestión urbana.

El concepto de "empresarialismo urbano" aborda los cambios en la forma de gestión urbana de los gobiernos locales (Harvey, 1989 y 2001; De Mattos, 2009; Cuenya y Corral, 2011), promoviendo la ampliación del rol de los gobiernos locales (fragmentación de la gestión urbana), junto con una redefinición en las prioridades de las políticas urbanas, reasignando un rol protagónico al capital privado en el desarrollo urbano. Así, la atracción de inversiones privadas se convirtió en un objetivo clave en la reestructuración urbana (Cuenya y Corra, 2011: 26).

El empresarialismo urbano expresa una nueva modalidad de gobernanza ${ }^{2}$, cuyas características principales son: 1) Alianzas entre el sector público y privado; 2) La ejecución y diseño de proyectos especulativos, y 3) Importancia en la economía política del lugar y no del territorio (Harvey, 2001: 373-374). Importante es no sobredimensionar el concepto como explicación de todas las transformaciones urbanas. "El poder para organizar el espacio deriva de todo un complejo de fuerzas movilizadas por diversos agentes sociales" (Harvey, 2001: 372), entendido como gobernanza, en la cual los gobiernos locales (urbanos) son sólo una parte, fungiendo en mayor o menor medida como coordinadores o facilitadores de procesos urbanos.

\subsection{Alianzas entre el sector público y privado}

Los distintos niveles de gobierno buscan generar las condiciones económicas propicias para atraer nuevas inversiones directas, esto es, a partir de diversos mecanismos. En primer lugar, incentivos financieros, como la extensión de derechos de construcción, e incentivos fiscales, como la desgravación impositiva de edificios protegidos. En segundo lugar, políticas de regulación y gestión favorables a los inversionistas, encaminadas hacia el uso del suelo, régimen de propiedad, entre otros

2 "La gobernanza hace alusión al hecho de que el poder para gobernar la ciudad excede al gobierno urbano; incluye una coalición amplia de fuerzas movilizadas por diversos actores sociales (empresas industriales, comercios, promotores inmobiliarios, instituciones educativas y religiosas, sindicatos, partidos políticos, organizaciones sociales, aparatos estatales)" (Cuenya y Corral, 2011: 27). 
-ejemplo de ello son los programas de subsidio para estimular la oferta y la demanda, los permisos de construcción de inmuebles de mayores alturas para aprovechar al máximo el uso de suelo, tal como sucede en el caso de la renovación urbana en el poniente de Santiago de Chile, en donde se construyen edificios de hasta veinte pisos que ofrecen vivienda subsidiada (Ducci, 2004: 139)-. Finalmente, la inversión pública directa, es decir, la participación de los distintos niveles de gobierno a través del mantenimiento del espacio público, mantenimiento de inmuebles de propiedad pública con valor patrimonial, así como mejoramiento y construcción de infraestructura de servicios básicos (agua, luz, drenaje).

Un ejemplo habitual de esta alianza es la reclasificación en el uso de terreno municipal en Baltimore en 1978, para el desarrollo privado de Harborplace (Plaza comercial -mall o shopping), el cual marca un punto de inflexión (Harvey, 2001: 374). Este modelo se reproduce en distintas ciudades: donación de un terreno de 6.500 metros cuadrados por parte del municipio de León (México) en 2011 a empresarios para la construcción de una universidad privada (La Jornada, 2011); El Ayuntamiento de Xinzo de Limia (Galicia, España) regalaría una parcela municipal de 74.000 metros cuadrados a la empresa privada Grupo ICG para la instalación del Centro Industrial Cárnico de Alta Tecnología (CICAT) (El País, 2012), por citar algunos casos cuya "facilitación" tiene como objetivo la reproducción del capital privado.

\subsection{Ejecución y diseño especulativo de proyectos}

En el empresarialismo urbano la ejecución y el diseño de proyectos urbanos suelen ser especulativos (Jaramillo, 2003), en contraste con la gestión gerencial que apostaba por el desarrollo racionalmente planeado y coordinado por las autoridades. Según De Mattos (2009), se ha impuesto la transición desde una gestión de tipo gerencial (la planificación) hacia otra de corte empresarialista (la gobernanza). En otras palabras, se ha pasado del gerencialismo que busca ante todo proveer con eficacia a todos los ciudadanos de los servicios de utilidad colectiva, al empresarialismo que se caracteriza netamente por una aproximación estratégica que busca el crecimiento económico, favorable a la toma de riesgo, a la innovación y propenso a orientarse hacia el sector privado, donde la competitividad pasa a ser el componente central de las estrategias respectivas.

Los proyectos urbanos suelen ser especulativos de dos maneras: a partir de la "especulación pasiva", también llamada "especulación del suelo", y de la "especulación inductiva" o "especulación inmobiliaria" (Jaramillo, 2003: 57-59). La primera es ejercida por los propietarios del suelo, que disponen o compran terrenos antes de que se generen los aumentos de precios y, sin realizar ninguna acción productiva, lo venden luego, apropiándose de las plusvalías. En los grandes proyectos, el primer rol corresponde típicamente al Estado nacional en tanto propietario mayoritario de grandes extensiones de tierras que quedaron desafectadas de sus usos previos (ferrocarriles y puertos) y que se ponen a la venta para usos jerárquicos (Cuenya y Corral, 2011: 29). La otra modalidad de especulación es ejercida por dos tipos de agentes: I) los promotores/desarrolladores que compran el suelo a precios de un uso que arroja rentas moderadas y luego de su acción transformadora venden el espacio construido con los precios que corresponden a ese nuevo uso jerarquizado, es decir, con rentas superiores (Jaramillo, 2003: 59), y II) el gobierno local, que invierte en obras de infraestructura para acondicionar el suelo y aprueba normativas asignando nuevos usos y coeficientes constructivos (Cuenya y Corral, 2011: 30). De esta manera, los distintos agentes van conformando proyectos de intervención urbana cuya forma de operar responde a procesos de especulación, permitidas por las autoridades locales. 
"En muchos casos, esto significa que el sector público asume el riesgo y el sector privado obtiene los beneficios" (Harvey, 2001: 374). En relación a lo anterior, en un estudio publicado por la Comisión Económica para América Latina (CEPAL) se analizan las políticas de concesiones viales en Chile, Colombia y Perú, en donde se implementan mecanismos para incorporar capitales privados mediante contratos de concesión de obra pública, empresas privadas que recuperan su inversión a través de las garantías del Estado, subsidios y peajes (Rufián, 2002: 12). Sin embargo, cada vez más, la absorción del riesgo recae en los gobiernos locales, como una de las características de la fragmentación de la administración pública que ha traído el proceso de descentralización.

\subsection{Economía política del lugar y no del territorio}

Este empresarialismo se centra más de cerca en la economía política del lugar y no del territorio, es decir, la mejora en las condiciones dentro de un lugar, como la imagen de la ciudad mediante la construcción de centros culturales, comerciales, de ocio o de oficinas, entre otros proyectos, dejando de lado la economía política del territorio, en el cual se contemplan los tipos de proyectos diseñados para mejorar las condiciones de vida y de trabajo dentro de una jurisdicción determinada (como vivienda y salud, por ejemplo). En este sentido, los proyectos de intervención tienen que ver con la mejora de un lugar, sin la menor preocupación por mejorar las condiciones de vida de la población, pudiendo traer como una de sus principales consecuencias los desplazamientos de población.

Estas tendencias que definen el empresarialismo urbano caracterizan muchas de las intervenciones urbanas que se han implementado en las últimas décadas y que en el discurso oficial reciben diversos nombres tales como "recuperación urbana", "revitalización", "regeneración" o "renovación", entre otros. Si el principio básico es crear una imagen urbana propicia para atraer inversión privada (definido en las tres tendencias que argumenta Harvey), este se concreta en los diversos proyectos urbanos cuyo objetivo es la reproducción del capital.

\subsection{Proceso de gentrificación}

Bajo el empresarialismo urbano se ha acelerado los procesos de transformación urbana en las áreas centrales, en particular el regreso del capital y de las clases medias, proceso conocido como gentrificación, el cual se ha producido en diversos momentos históricos, pero que actualmente prolifera en muchos centros urbanos de todo el mundo.

Más allá de las características que contemplan una gentrificación clásica o de las tendencias actuales del proceso de gentrificación en América Latina (Janoschka, Sequera y Salinas, 2013; Salinas, 2013), es importante destacar el elemento clave que motivó el uso del concepto, que hace referencia a un problema social y/o de clase (Slater, 2006: 752; Slater, 2008: 216; Smith, 2008: 33). Estando de acuerdo con Less, Slater y Wyly (2008: 158), se consideran cuatro elementos a para identificar un proceso de gentrificación: (I) reinversión de capital, (II) mejoramiento de las condiciones sociales por la entrada de grupos de mayores ingresos ${ }^{3}$, (III) cambios en el paisaje urbano y (IV) desplazamiento directo o indirecto de grupos de ingresos menores.

3 El desplazamiento por un sector de la población de mayores ingresos no implica necesariamente sólo a sectores de clase alta, como se caracterizó el proceso en sus orígenes, puesto que "dejó de constituir un asunto exclusivo de las clases altas (...), y se convirtió en una seria opción residencial también para lo más granado de la clase media." (Smith, 2001: 19). 
Destacando estas cuatro características del proceso de gentrificación, resulta de interés para la presente investigación resaltar la gestión empresarialista por parte del gobierno del Distrito Federal y del gobierno de la Delegación (municipio) Cuauhtémoc, quienes han generado las condiciones económicas propicias para la inversión de capital y su transformación del territorio de las colonias céntricas de la ciudad.

\section{Transformaciones urbanas en áreas centrales de la ciudad de México}

La ciudad de México, incluyendo su zona metropolitana ${ }^{4}$, registró 20.116 .842 de habitantes en el Censo de Población y Vivienda, 2010 del Instituto Nacional de Estadística y Geografía (INEGI), lo que representa casi el 18 por ciento de la población total del país. Esta concentración de población se produjo hacia la segunda mitad del siglo XX al pasar de 2.952.000 de habitantes en 1950 a 8.623.000 en 1970 y 15.048 .000 en 1990. Así, respecto del crecimiento de la ciudad de México se pueden diferenciar dos claras tendencias. Por un lado, un freno del ritmo de crecimiento en las Delegaciones del Distrito Federal (la población pasó de 8.831.079 en 1980 a 8.851.080 en 2010), e incluso una pérdida de población en el área central (Delegaciones: Cuauhtémoc, Benito Juárez, Venustiano Carranza y Miguel Hidalgo) al pasar de 2.902.969 en 1970 a 1.721 .137 en 2010). Y por otro lado, un crecimiento constante de la periferia urbana (ver Aguilar, 2002), concentrando los municipios metropolitanos de la ciudad de México el 56 por ciento de la población.

La pérdida de población del área central está relacionada con el proceso de recentralización con desconcentración, en el cual se aprecia una desconcentración de la actividad industrial hacia los municipios periféricos, pérdida de uso de suelo habitacional, así como a la relocalización de espacios recreativos, de ocio y servicios educativos. Esta situación, aunada al seísmo de 1985, provocó un importante deterioro físico y funcional de los inmuebles localizados en del área central. Al mismo tiempo, la recentralización de actividades del sector terciario localizó gran parte de servicios al productor y al consumidor en el poniente del área central, en particular a partir de la Alameda Central hasta la zona de Polanco, constituyendo la Avenida Reforma como uno de los corredores más importantes en cuanto a la localización de oficinas y servicios de la ciudad de México. Esta situación revalorizó las áreas aledañas, siendo la colonia Condesa un caso paradigmático.

\subsection{Transformaciones en la colonia Condesa}

La colonia Condesa se localiza en la delegación Cuauhtémoc, al poniente del centro histórico (Figura 1), conocida por sus áreas verdes y zona gastronómica es en la actualidad un referente de la Ciudad de México.

Esta colonia ha sufrido grandes transformaciones a partir de los años noventa, las cuales podemos sintetizar en: proliferación de establecimientos comerciales y de oferta inmobiliaria de uso habitacional y cambios en la composición sociodemográfica de los residentes. Estas son características del proceso de gentrificación que está viviendo dicho barrio, propiciado por una gestión empresarialista del gobierno de la Ciudad de México (principalmente mediante la Secretaría de Desarrollo Urbano y Vivienda del Gobierno del Distrito Federal) y del gobierno de la Delegación Cuau-

4 La Zona Metropolitana del Valle de México está conformada por 58 municipios del estado de México, un municipio del estado de Hidalgo y 16 Delegaciones del Distrito Federal, una superficie aproximada de $7.866,1 \mathrm{~km}^{2}$. 
htémoc (instancia responsable de otorgar licencias de apertura de establecimientos comerciales y licencias de construcción/edificación).
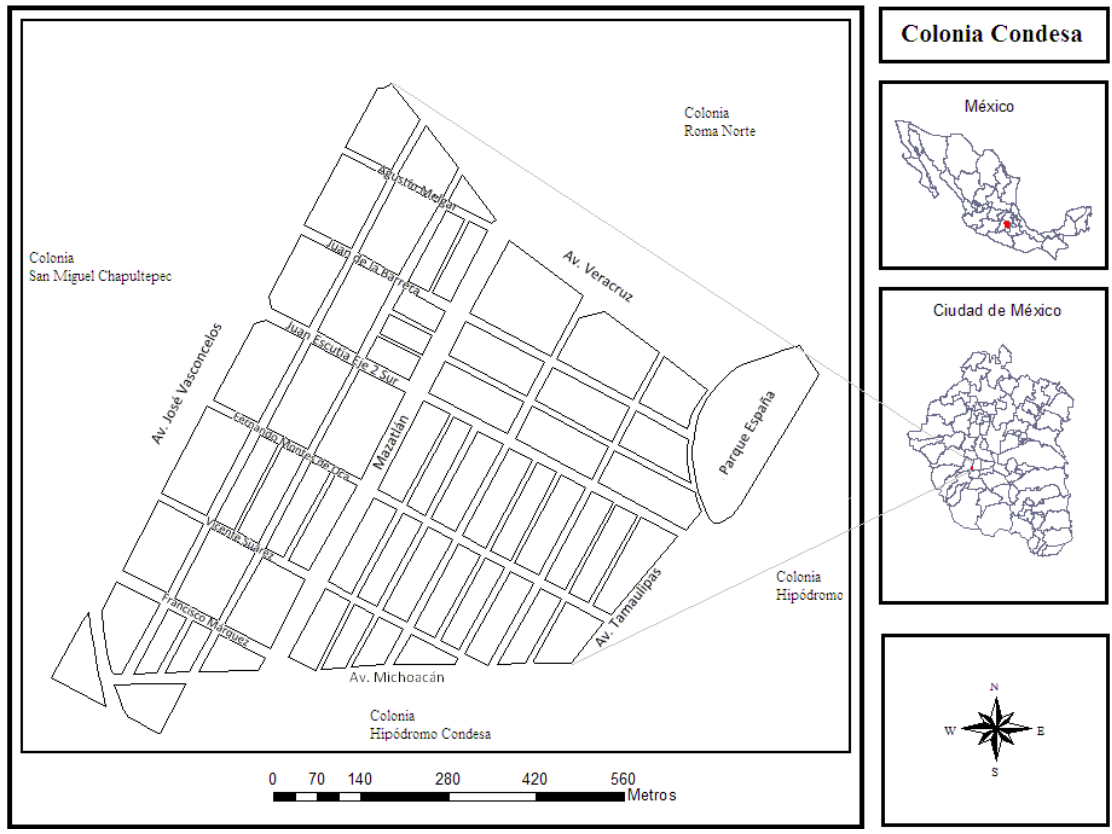

Figura 1. Colonia Condesa.

Fuente: Elaboración propia a partir de información cartográfica de INEGI, 2013.

\subsection{Gobierno local e Incremento de establecimientos comerciales y oferta inmobiliaria}

A principios de los noventa, comienzan a establecerse negocios y oficinas en la colonia Condesa mediante dos mecanismos: cambio de uso de suelo, de habitacional a comercial y oficinas, y cambio de giro mercantil, el cual implica que aquellos establecimientos de bajo impacto (tienda de abarrotes, reparación de calzado, panadería, etcétera) cambien su giro a alto impacto (establecimientos comerciales con venta de bebidas alcohólicas como restaurantes, bares y centros nocturnos).

En lo que tiene que ver con cambios de uso de suelo, en primer lugar, hay establecimientos comerciales y oficinas que están operando con certificados de cambio de uso de suelo emitidos por la Secretaría de Desarrollo Urbano y Vivienda (SEDUVI), es decir, con funcionamiento legal. Cabe mencionar que esta forma de cambios de uso de suelo han sido pocos: de un total de 800 cambios de uso de suelo que se dieron en el Distrito Federal de 1998 al 2012, 63 corresponden a la Delegación Cuauhtémoc, y de estos, 11 corresponden a predios localizados en la Condesa (Oficina de Información Pública, SEDUVI). No obstante, en segundo lugar, también hay establecimientos comerciales y oficinas en diversos predios que han cambiado su antiguo uso habitacional al margen de la ley ${ }^{5}$. Respecto de estos últimos, su actual funcionamiento es posible gracias a tres prácticas permitidas y toleradas por los gobiernos locales, los cuales son: derechos adquiridos, recurso de amparo y corrupción.

5 El Plan General de Desarrollo Urbano del Distrito Federal (2003: 34) reconoce que la ilegalidad ha sido la vía más usada en los cambios de uso de suelo. 
Los "derechos adquiridos" 6 son documentos que demuestran que su establecimiento mercantil ha funcionado desde antes de la zonificación del Plan Parcial de Desarrollo Urbano de 1982 o de las modificaciones contempladas en los programas de desarrollo delegacional vigente; el recurso de "amparo" es un recurso emitido ante un ministerio público que posibilita su funcionamiento, y las prácticas de corrupción se concretan por medio de un pago mensual de los comerciantes a las autoridades locales. Estas características forman parte de la gestión empresarialista del gobierno local, permitiendo que continúen operando las actividades comerciales en la colonia, por ello, la zonificación actual de uso de suelo realizado por las autoridades encargadas al respecto -SEDUVI- (Figura 2), dista mucho del uso de suelo real (Figura 3).
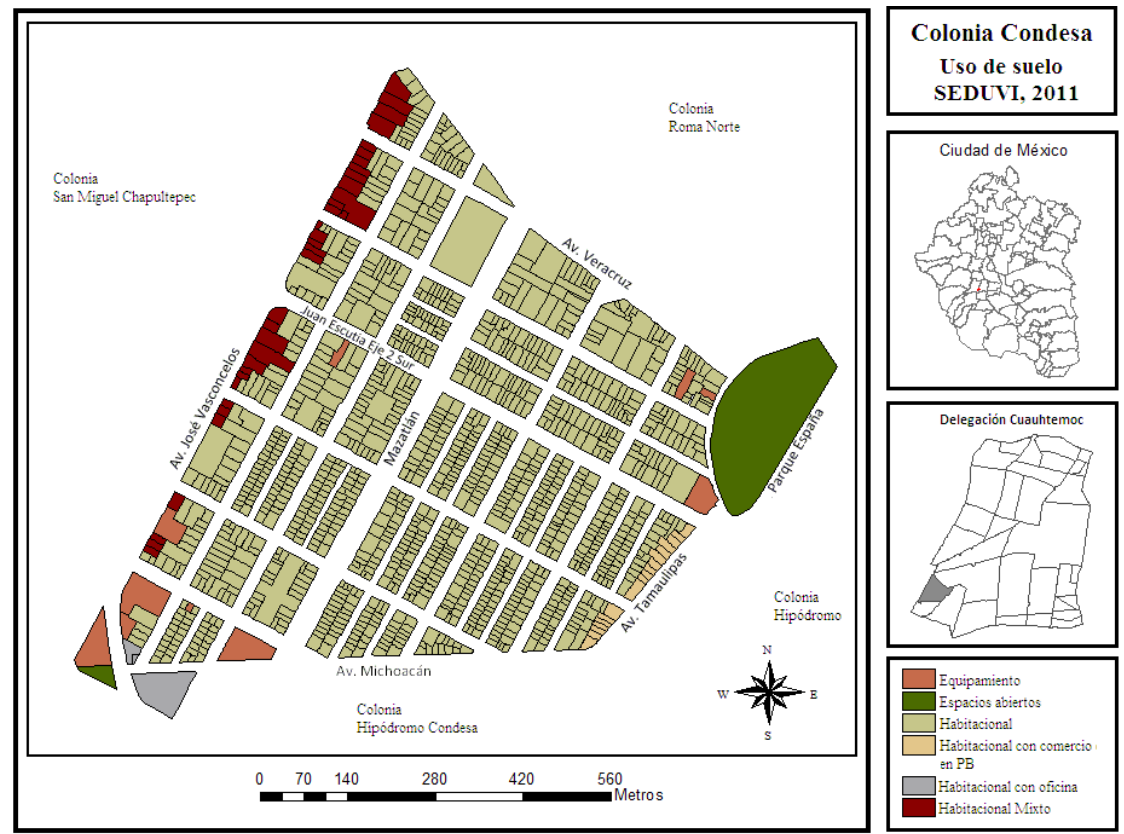

Figura 2. Uso de suelo SEDUVI, 2011.

Fuente: Elaboración propia a partir de datos disponibles en el Sistema de Información Geográfico de la Secretaría de Desarrollo Urbano y Vivienda del Distrito Federal.

Los cambios de giro mercantil es otra forma en que se ha transformado la actividad comercial, impactando en las condiciones económicas y sociales de los residentes de la Condesa. Tanto los cambios de giro mercantil como los cambios de uso de suelo, dentro y fuera de la ley, han favorecido la presencia de 1.009 establecimientos comerciales con giro de oficinas y/o empresas registradas en el Padrón de Establecimientos Comerciales, de las cuales 122 corresponden a giros de alto impacto ${ }^{7}$.

6 Se entenderá por Certificado de Acreditación de Uso del Suelo por Derechos Adquiridos el documento público que tiene por objeto reconocer los derechos de uso del suelo y superficie que por el aprovechamiento legítimo y continuo tienen los propietarios, poseedores o causahabientes de un bien inmueble (Ley de Desarrollo Urbano del Distrito Federal, 2010: 35).

7 Información solicitada por el autor y recibida el 22 de junio de 2011 por el Jefe de Unidad de Departamento de Giros Mercantiles (comerciales) de la Delegación Cuauhtémoc, Lic. Luis Mauricio López Ruiz. 

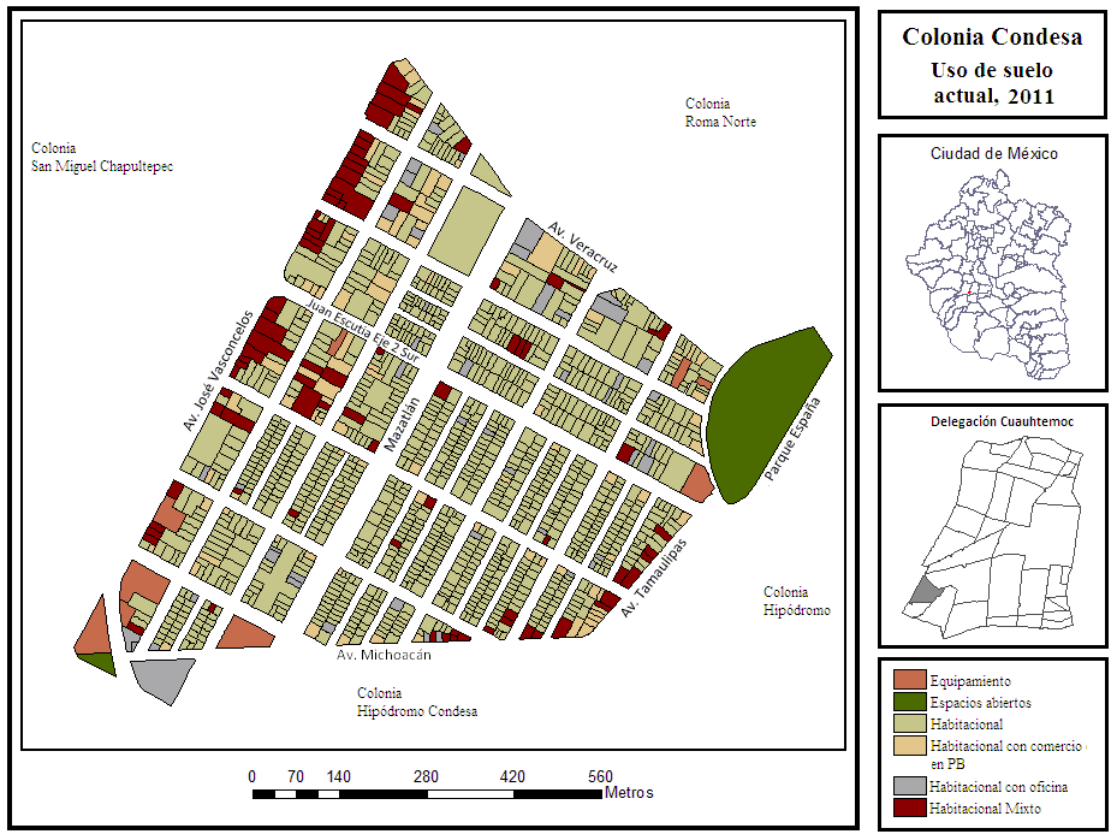

Figura 3. Uso de suelo "actual", 2011.

Fuente: Elaboración propia a partir de información obtenida mediante trabajo de campo.

Estos cambios de uso de suelo y giro mercantil, dentro y fuera de la ley, que se están realizando en la colonia, forman parte de una visión por parte de las autoridades locales de hacer de la Condesa un atractivo turístico, un espacio para el retorno del capital y de la clase media. Además, se ha posibilitado el desarrollo de diversos proyectos inmobiliarios de varios pisos de construcción destinados para uso habitacional, dirigidos a población de clase media y alta.

Si bien es cierto que el sector inmobiliario ha sido muy dinámico en los últimos años, en general en toda la Ciudad de México (30 por ciento del mercado nacional total de vivienda nueva terminada), la colonia Condesa, con un 3,5 por ciento, es la colonia de mayor oferta de la delegación Cuauhtémoc (Cuadro 1).

\begin{tabular}{|cc|c|}
\hline Colonia & (Delegación) & $\%$ \\
\hline Bosques de las Lomas & (Miguel Hidalgo) & 13,2 \\
\hline Santa Fe & (Álvaro Obregón) & 8,6 \\
\hline Polanco Chapultepec & (Miguel Hidalgo) & 8,2 \\
\hline Del Valle & (Benito Juárez) & 7,9 \\
\hline Lomas de Chapultepec & (Miguel Hidalgo) & 7,3 \\
\hline Bosques de las Lomas & (Cuajimalpa) & 6,5 \\
\hline Jesús del Monte & (Cuajimalpa) & 5,1 \\
\hline Condesa & (Cuauhtémoc) & 3,5 \\
\hline Cuajimalpa & (Cuajimalpa) & 3,0 \\
\hline Porcentaje por cada delegación & $\mathbf{1 0 0}$ \\
\hline
\end{tabular}

Cuadro 1. Colonias con la mayor oferta de vivienda del Distrito Federal, 2011.

Fuente: Paredes, 2011 con modificaciones propias. 
Sin embargo, el lugar destacado que ocupa la colonia Condesa no se refiere únicamente a la cantidad de oferta de vivienda nueva o usada, pues no es comparable con los grandes desarrollos habitacionales que se realizan en la periferia de la ciudad de México. Su importancia principal radica en que esa oferta se dirige a un sector de la población de ingresos medios y altos. Puesto que forma parte de las cuatro colonias con mayor aumento del precio de las viviendas de la delegación Cuauhtémoc, después de colonias como Cuauhtémoc, Hipódromo Condesa y Lindavista, en donde se ha registrado un incremento de plusvalía del orden del 24,3 por ciento, 22,3 por ciento y 16,9 por ciento respectivamente, mientras que la colonia Condesa ha incrementado 14,2 por ciento (Paredes, 2011).

Los nuevos desarrollos de vivienda son construcciones verticales que buscan aprovechar hasta el último metro cuadrado disponible, enfocándose en los grandes espacios que ocupan las viviendas propiedad de personas mayores que están migrando a otras zonas más tranquilas en la ciudad. La oferta de departamentos en la colonia Condesa hasta el mes de junio de 2012 era de 524 departamentos, de los cuales 224 estaban en construcción. Estos se encuentran en tres de los principales proyectos de departamentos a cargo de la inmobiliaria Baita Promoción S.A. de C.V, inmobiliaria Casa Carreto Bienes Raíces e inmobiliaria JSa Diseño Desarrollo. En el primer proyecto se comercializan departamentos nuevos desde 45,3 $\mathrm{m}^{2}$ (según el Plan Delegacional de Desarrollo Urbano de la Delegación Cuahtémoc 2008, vigente, la vivienda mínima en esta zona habitacional es de $90 \mathrm{~m} 2$ ) hasta $172 \mathrm{~m}^{2}$ cuyo valor va de 129.600 dólares a 557.800 dólares respectivamente, ofreciendo un total de 180 departamentos. En el segundo proyecto se manejan 34 departamentos de 84 $\mathrm{m}^{2}$ cuyo precio es de 230.860 dólares. Y, en el tercer proyecto, se ofrecen 10 departamentos, cuyo valor va desde 494.110 dólares de $133 \mathrm{~m}^{2}$ hasta 639.910 dólares de $174 \mathrm{~m}^{2}$. Cabe mencionar que hacia el primer semestre del 2012, estos tres proyectos presentan demandas ante la Procuraduría Ambiental y de Ordenamiento Territorial del Distrito Federal (folios: 2011/786/SOT391, 2011/2103/SOT968 y 2011/786/ SOT391, respectivamente) por rebasar los niveles de altura permitidos que establece la Secretaría de Desarrollo Urbano y Vivienda.

La oferta de vivienda presenta las siguientes características: construcciones verticales que sobrepasan los 3 niveles permitidos por las autoridades locales, viviendas por debajo del límite de $90 \mathrm{~m}^{2}$ de construcción, desarrollos sobre inmuebles catalogados por el Instituto Nacional de Bellas Artes (INBA) y departamentos dirigidos a un sector de la población de ingresos medios y altos.

De esta manera, la actividad económica que está transformando a la colonia condesa tiene que ver con cambios de uso de suelo, cambios de giro mercantil y crecimiento de desarrollos habitacionales. Esto se produce en un contexto de gestión empresarialista por parte del gobierno local, cuya prioridad es la economía del lugar y no del territorio, estimulando la inversión en lugares (restaurantes, bares, desarrollos residenciales) para hacer de esta colonia más competitiva en la búsqueda de inversión privada.

\subsection{Cambios demográficos en la Condesa}

La colonia Condesa no escapa de la tendencia a la pérdida de población que ha venido sufriendo el área central a lo largo de los últimos 40 años. Sin embargo, a diferencia de otras zonas, el declive demográfico responde a cambios en la conformación del número de integrantes por vivienda y a movimientos de población relacionados a las transformaciones recientes. 
Según los Censos de Población y Vivienda de 1990 y 2010 del INEGI, la colonia Condesa pasó de una población de 15.916 a 11.797, es decir, una pérdida del 25,88 por ciento de la población en dicho periodo. Una pérdida muy sensible si la comparamos con la pérdida de población de toda la delegación Cuauhtémoc que fue de 10,76 por ciento.

Siendo una colonia con los atributos urbanos antes mencionados (localización, accesibilidad, patrimonio artístico y espacios públicos), lo que la convierten en una zona atractiva, habría que responder ¿por qué continúa o por qué se registra esta pérdida de población? Para contestar parte de esta interrogante, empecemos por interpretar los datos que presenta el INEGI en diversos años.

Si analizamos las variaciones de población en los censos por rangos de edades encontramos que la pérdida de población más sensible se observa de los 0 a los 24 años, la cual disminuyó en 38,44 por ciento, mientras que el rango de edad en el que se muestra una tendencia contraria a la pérdida de población es de 25 a 59 años de edad, la cual pasó de un porcentaje 54,29 por ciento a un 62,39 por ciento. En términos absolutos, este grupo de edad pasó de 7.144 a 7.360 habitantes, lo que representa un incremento del 3,02 por ciento. Por esta razón se evidencia que la mayor cantidad de población que emigra se encuentra en el rango de edad de los 0 a los 24 años, mientras que la población que permanece e incluso la nueva población que llega se encuentra en el rango de 25 a 59 años, la cual ha incrementado los índices socioeconómicos como alfabetización e instrucción superior. Es decir, características de un sector de la población de clase media y alta que está ocupado la colonia Condesa.

Esta colonia presenta los menores porcentajes de analfabetismo de la población de 15 años y más en la delegación Cuauhtémoc con un 0,53 por ciento, muy por arriba del promedio delegacional que fue de 2,83 por ciento. Otro dato al respecto es el incremento de la población de 18 años y más con educación superior, que pasa de 37,71 por ciento a 47,70 por ciento en el periodo de 1990 a 2000, únicamente por debajo de la colonia Cuauhtémoc, la cual muestra los mayores porcentajes de población con educación superior en la delegación de igual nombre, con 48,59 por ciento. Por su parte, el grado promedio de escolaridad ascendió de 12,20 a 14,06 por ciento, del año 2000 al 2010, también por encima de los indicadores de otras colonias y del promedio delegacional, que fue de 12,47 por ciento.

Por otra parte, para el año 2010, de un total de 5.350 viviendas particulares tan sólo 4.508 viviendas estaban habitadas, es decir, un 15,74 por ciento de las viviendas están deshabitadas o, lo que es más probable, se utilizan como oficinas o comercio, siendo este un elemento que caracteriza el irregular uso de suelo que se ha propiciado en los últimos años.

Además de haber disminuido el uso de suelo habitacional por el incremento de oficinas y comercios, también ha disminuido el número de ocupantes por vivienda: el promedio, según los censos de 1990, 2000 y 2010, ha disminuido de 3,2 y 2,8 hasta 2,4 por ciento, respectivamente, por encima del promedio delegacional, el cual pasó de 3,6 y 3,3 hasta 2,9 por ciento.

De esta manera, podemos apreciar a partir de diversos indicadores socioeconómicos, los cambios que caracterizan a la población de la colonia. En este sentido, se están produciendo cambios importantes en la composición sociodemográfica, derivados de la llegada de nuevos residentes de mayores ingresos y con mayor capital cultural, quienes han reducido el promedio de habitantes por vivienda. Esta situación 
así como los cambios de uso de suelo al margen de la ley explican la aparente pérdida de población.

\section{Conclusiones}

El empresarialismo urbano, como forma de gestión urbana en el contexto neoliberal, ha sido adoptado por gobiernos locales de diversas ciudades, para afrontar problemas económicos y presupuestales de la administración pública. Se pretende estrechar alianzas entre el sector público y privado, con el fin de estimular la inversión de capital privado. Distintas son las estrategias que caracterizan la gestión empresarialista destacando de esta alianza del sector público y privado, la cesión o donación de activos públicos al sector privado, proyectos urbanos de ejecución y diseño especulativo y la imposición de la economía política del lugar y no del territorio (Harvey, 2001).

En lo que a la ciudad de México se refiere, tal como se analizó a lo largo del texto, las estrategias empresarialistas giran en torno a resaltar la importancia del lugar, promoviendo la inversión en espacios selectivos, como lo ha sido la Alameda Central, Av. de la Reforma y distintos barrios del área central. En nuestro caso de estudio, el empresarialismo urbano se ha caracterizado por: a) autorización de cambios de uso de suelo, permisos de apertura de negocios y otorgamiento de licencias de funcionamientos permitiendo el cambio de giro mercantil de los establecimientos comerciales de bajo impacto a alto impacto; b) ausencia de control y verificación de funcionamiento acorde a los distintos reglamentos vigentes; y c) distintas intervenciones en espacios abiertos, como el mantenimiento del parque España y los camellones de la calle Mazatlán, mejoramiento del alumbrado público, desplazamiento de vendedores ambulantes y fuerte presencia de seguridad pública. Todo ello, con la intención de generar las condiciones propicias para la inversión privada.

Esta situación ha propiciado diversas transformaciones urbanas, incrementando los establecimientos comerciales, la oferta de vivienda para población de ingresos medios y altos, cambios sociodemográficos y desplazamiento de población, elementos que caracterizan un proceso de gentrificación.

Este caso de estudio, muestra la importancia de discutir procesos de transformación urbana en el contexto del empresarialismo urbano, el cual conforma una tendencia que está inmersa en distintas transformaciones urbanas que caracterizan a la ciudad neoliberal.

\section{Bibliografía}

Aguilar, A. (2002). Las mega-ciudades y las periferias expandidas. Ampliando el concepto de Ciudad de México. EURE, Revista de Estudios Urbano y Regionales, v. 28, n.85, Santiago de Chile. DOI: http://dx.doi.org/10.4067/S0250-71612002008500007.

Amin, A. y Robins, K. (1994). El retorno de las economías regionales. Geografía mítica de la acumulación flexible. En Benko, G. y Lipietz, A., Las regiones que ganan. Distritos y redes. Los nuevos paradigmas de la geografía económica. Valencia: Ediciones Alfons El Megnánim. 
Boyer, R. (1994). Las alternativas al fordismo de los años 80 al siglo XXI. En: Benko, G. y Lipietz A., Las regiones que ganan: Distritos y redes. Los nuevos paradigmas de la geografía económica. Valencia: Ediciones Alfons El Megnánim.

Carrión, F. (2004). Los centros históricos en la era digital. Icono, Revista de Ciencias Sociales, septiembre, $\mathrm{n}^{\circ}$ 20, 35-44. DOI: http://dx.doi.org/10.17141/iconos.20.2004.60.

Ciccolella, P. y Mignaqui, I. (2009). Capitalismo global y transformaciones metropolitanas: enfoques e instrumentos para repensar el desarrollo urbano. En Poggiese, $\mathrm{H}$. y Cohen, T. (comp.), Otro desarrollo urbano: ciudad incluyente, justicia social y gestión democrática, 1a ed. Buenos Aires: Consejo Latinoamericano de Ciencias Sociales (CLACSO).

Cuenya, B., Corral, M. (2011). Empresarialismo, economía del suelo y grandes proyectos urbanos: el modelo de Puerto Madero en Buenos Aires. EURE, Revista de Estudios Urbano y Regionales, v. 37, $\mathrm{n}^{\circ}$ 111. DOI: http://dx.doi.org/10.4067/ S0250-71612011000200002.

De Mattos, C. (2002). Redes, nodos y ciudades: Transformación de la metrópoli latinoamericana. Santiago de Chile: Instituto de Estudios Urbanos y Territoriales, Pontificia Universidad Católica de Chile.

De Mattos, C. (2009). Modernización capitalista, Metamorfosis Urbana y Competitividad en América Latina. Ponencia presentada en Seminario Internacional La Investigación Urbana: Perspectivas y Desafíos. Recuperado de http://www.uarm.edu.pe/Docs/ investigacion/doc/competitividad_mattos.

Ducci, M. (2004). Las batallas urbanas de principios del tercer milenio. En De Mattos, C. et. al., Santiago en la globalización: ¿Una nueva ciudad? Santiago de Chile: Ediciones SUR y EURE Libros.

Duhau, E. y Giglia A. (2008). Las reglas del desorden: habitar la metrópoli. México: UAM-A y Siglo XXI.

El País (2012). La Xunta beneficia un proyecto empresarial de un exalcalde de Xinzo, 1 de junio. Recuperado de http://ccaa.elpais.com/ccaa/2012/06/01/galicia/1338579697_348968.html.

Gaceta Oficial del Distrito Federal (2010). Ley de Desarrollo Urbano del Distrito Federal, 15 de julio. Recuperado de http://www.aldf.gob.mx/archivo-2ec5c052a850fa2a8491ca817aee8439.pdf.

Goulart, R. (2005). O processo de reabilitação e renovação urbana na cidade do Rio de Janeiro e suas perspectivas. Scripta Nova. Revista electrónica de geografía y ciencias sociales. Barcelona: Universidad de Barcelona, 1 de agosto, v. IX, n 194 (44). Recuperado de http://www.ub.es/geocrit/sn/sn-194-44.htm.

Harvey, D. (1989). From managerialism to entrepreneurialism: The transformation in urban governance in late capitalism. Geografiska Annaler. Series B, Human Geography, v. 71, n 1, 3-17. Recuperado de http://dx.doi.org/10.2307/490503.

Harvey, D. (1990). La condición de la posmodernidad. Buenos Aires: Amorrortu.

Harvey, D. (2001). Espacios del capital. Hacia una geografía crítica. Madrid: Akal.

Herzog, L. (2004). Globalización, política y revitalización del centro histórico de la ciudad de México". En Rodríguez, A. y Tamayo, S. (coord), Los últimos cien años, los próximos cien..., México: Cultura Universitaria, Serie ensayo, $n^{\circ} 73$. 
Hiernaux, D. (1998). Reestructuración económica y cambios territoriales en México. Un balance 1982-1995. En De Mattos, C. (comp.), Globalización y territorio impactos y perspectivas. Santiago de Chile: Universidad Católica de Chile y Fondo de Cultura Económica.

Janoschka M.; Sequera, J. y Salinas, L. (2013). Gentrification in Spain and Latin America - a Critical Dialogue. International Journal of Urban and Regional Research, v. 37, septiembre. DOI: 10.1111/1468-2427.12030.

Jaramillo, S. (2003). Los fundamentos económicos de la Participación en Plusvalías. Bogotá y Cambridge: Ed. Universidad de los Andes y Lincoln Institute of Land Policy.

La Jornada (2011). En menos de dos años, el gobierno de León regaló doce predios al clero y a organizaciones privadas, 25 de septiembre. Recuperado de http://www.jornada.unam.mx/2011/09/25/estados/030n2est.

Lees, L.; Slater, T. y Wyly, E. (2008). Gentrification. New York: Routledge.

Méndez, R. (1997). Geografía económica: la lógica del capitalismo global. Barcelona: Ariel.

Monroy, R. y Martínez, S. (2008). Capital inmobiliario y transformación del territorio. Ciudades, Análisis de la coyuntura, teoría e historia urbana, $n^{\circ} 79$, julio-septiembre, Puebla, México.

Mertins, G. (2005). Transformaciones recientes en las metrópolis latinoamericanas y repercusiones espaciales. En Welch, M. (ed.), Buenos Aires a la deriva. Transformaciones urbanas recientes. Buenos Aires: Editorial Biblos.

Paredes, M. (2011). Guía de precios DF 2011. Metroscubicos, 6 de abril. Recuperado de http://www.metroscubicos.com/precios.m3/colima/guia_de_precios_df2011/pagina1.

Portes, A. y Roberts, B. (2008).Introducción. La ciudad bajo el libre mercado. La urbanización en América Latina durante los años del experimento neoliberal”. En Portes, A. et. al. (ed.), Ciudades Latinoamericanas. Un análisis comparativo en el umbral del nuevo siglo. Buenos Aires: Prometeo Libros.

Pradilla, E. (2009). Los territorios del neoliberalismo en América Latina. México: UAM y Miguel Ángel Porrúa.

Ramírez, J. y Hauser, A. (1996). El concepto de localización en los sistemas rígidos y flexibles de producción. CIDE, $n^{\circ} 55$, México, D.F.

Rufián, D. (2002). Políticas de concesión vial: análisis de las experiencias de Chile, Colombia y Perú. Santiago de Chile: CEPA.

Salinas, L. (2013). Gentrificación en la ciudad latinoamericana. El caso de Buenos Aires y Ciudad de México. GeoGraphos. Alicante: Grupo Interdisciplinario de Estudios Críticos y de América Latina (GIECRYAL) de la Universidad de Alicante, v. 4, n 44, 281 305. DOI: 10.14198/GEOGRA2013.4.44.

Salinas, L. (2014). Gentrificación en el área central de la Ciudad de México: El caso de la colonia Condesa. En Janoschka, M. y Hidalgo, R. (eds.), Ciudad neoliberal: Crisis, Resistencias y Escenarios de futuro. Santiago de Chile: Pontificia Universidad Católica de Chile, serie GEOLibros (179-198).

Slater, T. (2006). The Eviction of Critical Perspectives from Gentrification Research. International Journal of Urban and Regional Research, v. 30 (4), 737-757. DOI:10.1111/j.1468-2427.2006.00689.x. 
Slater, T. (2008). A Literal Necessity to be Re-Placed: A Rejoinder to the Gentrification Debate. International Journal of Urban and Regional Research, v.32 (1), 212-223. DOI: 10.1111/j.1468-2427.2008.00781.x.

Smith, N. (2001). Nuevo globalismo, nuevo urbanismo. Documents dAnálisi Geográfica, $n^{\circ} 38,15-32$.

Smith, N. (2008). Gentrificación generalizada: de la anomalía local a la "regeneración urbana" como estrategia global urbana. En Diputació de Barcelona, Ciudades en (re) construcción: necesidades sociales, transformación y mejora de barrios (31-48). Barcelona: Àrea de Presidència. Observatori Territorial, Diputació de Barcelona. 